\title{
Early and late outcomes with prolonged open chest management after cardiac surgery
}

Joshua K. Wong, MD, Devang J. Joshi, MD, Amber L. Melvin, MD, Christopher T. Aquina, MD, MPH, William J. Archibald, BS, Alcina K. Lidder, BA, Christian P. Probst, MD, MPH, Howard T. Massey, MD, George L. Hicks, MD, and Peter A. Knight, MD

\begin{abstract}
Objectives: Open chest management (OCM) is an important intervention for patients who are unable to undergo sternal closure after cardiac surgery. This study reviews the factors associated with a prolonged need for this intervention and investigates its association with early and late mortality.

Methods: Patients undergoing OCM from January 2009 to December 2014 were reviewed. Differences in the median duration of OCM when a perioperative variable was present versus its absence were determined and variables significant at $P \leq .1$ were analyzed using Poisson regression for factors associated with prolonged OCM. Multivariable logistic regression and Cox proportional hazards models were developed to investigate perioperative factors that were associated with early and late mortality.
\end{abstract}

Results: A total of 201 patients $(5 \%)$ required OCM and the overall median duration of this intervention was 3 days. The use a temporary assist device (median, 7 vs 2 days; $P<.001$ ), pneumonias (median, 11 vs 3 days; $P<.001$ ), sternal re-explorations (median, 6 vs 2 days; $P<.001$ ), and renal failure (median, 6 vs 3 days; $P=.02$ ) were among the factors that were highly associated with prolonged OCM using Poisson regression. Thirty-day mortalities occurred in 32 patients $(16 \%)$ and were significantly associated with emergency surgery $(P=.03)$, sternal re-explorations $(P=.001)$, and OCM duration (median, 6 vs 3 days; $P=.02$ ). On multivariable logistic regression and Cox analysis, delaying sternal closure by 1-day increments increased the risk of early and late mortality by $11 \%(P=.01)$, and $9 \%(P<.001)$, respectively.

Conclusions: Prolonged OCM was associated with increasing perioperative morbidity and a higher risk of early and late mortality. ( $\mathrm{J}$ Thorac Cardiovasc Surg 2017;154:915-24)

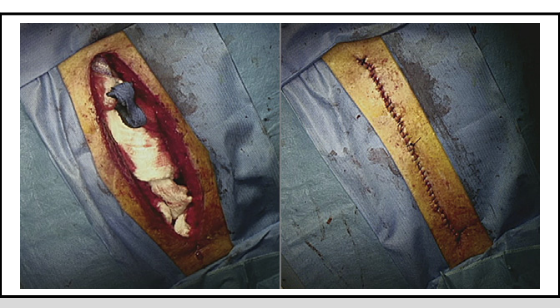

Open chest management technique for mediastinal structure isolation and protection.

Central Message

Prolonged open chest management is associated with increased patient morbidity and a higher likelihood of early and late mortality.

\section{Perspective}

An increased complication rate in patients who require prolonged open chest management emphasizes the importance of avoiding delays to early sternal closure to reduce the risk of additional morbidity in this critically ill patient population. The increased risk of early and late mortality for each day sternal closure is delayed by $9 \%$ and $11 \%$, respectively, provides further evidence to support this approach.

See Editorial Commentary page 925.
Postcardiotomy open chest management (OCM) for the treatment of mediastinal edema was first described by Riahi and colleagues, ${ }^{1}$ who used a novel technique of sternal traction using an overhead pulley to relieve cardiac compression and improve patient hemodynamic parameters. As a result of improving perioperative

\footnotetext{
From the Division of Cardiac Surgery, University of Rochester Medical Center, Rochester, NY

Read at the 96th Annual Meeting of The American Association for Thoracic Surgery, Baltimore, Maryland, May 14-18, 2016.

Received for publication May 25, 2016; revisions received Feb 16, 2017; accepted for publication March 20, 2017; available ahead of print June 1, 2017.

Address for reprints: Joshua K. Wong, MD, Division of Cardiac Surgery, University of Rochester Medical Center, 601 Elmwood Ave, Box SURG, Rochester, NY 14642 (E-mail: Joshua_Wong@urmc.rochester.edu). $0022-5223 / \$ 36.00$

Copyright (c) 2017 by The American Association for Thoracic Surgery http://dx.doi.org/10.1016/j.jtcvs.2017.03.143
}

critical care with a resultant increase in survival rates, ${ }^{2-6}$ and a reported low incidence of mediastinal infections, indications for this technique have expanded to include the treatment of severe coagulopathy, hemodynamic instability, intractable arrhythmias, and the insertion of temporary assist devices, ${ }^{3-5,8,9}$ establishing it as an important treatment option for patients who are unable to undergo primary sternal closure after cardiac surgery.

Scanning this QR code will take you to a supplemental video. To view the AATS 2016 Webcast, see the URL next to the webcast thumbnail. 


\section{Abbreviations and Acronyms \\ $\mathrm{CPR}=$ cardiopulmonary resuscitation \\ DSC $=$ delayed sternal closure \\ DSWI $=$ deep sternal wound infection \\ LVAD $=$ left ventricular assist device \\ $\mathrm{LVEF}=$ left ventricular ejection fraction \\ OCM $=$ open chest management}

The need for OCM occurs in $1 \%$ to $4 \%$ of general adult cardiac surgery and has been reported to be required in up to $50 \%$ of left ventricular assist device (LVAD) implants in a recent multi-institutional study. ${ }^{7}$ Despite the established use of OCM in the current era, the perioperative factors that are associated with a prolonged need for this intervention have not been described to date. Knowledge of these factors may be helpful in the identification of certain high-risk characteristics for needing longer OCM, allowing earlier interventions for risk-factor modification and could also prove valuable in providing clinicians with a better understanding on the natural history of complications that develop while the chest is open. It also remains unclear if maintaining OCM for a prolonged duration is associated with any significant effect on early or late mortality. The results from studies in the literature have indicated that there may be a relationship between a prolonged time to delayed sternal closure (DSC) and poorer patient outcomes ${ }^{2,3}$; however, a clearer understanding of this association will be important, particularly during the risk-benefit assessment when deciding appropriate timing of sternal closure.

We report a comprehensive review of a large series of patients undergoing OCM at a quaternary academic medical institution. In addition, we present an analysis of the perioperative factors associated with the need for prolonged OCM and investigate whether such a need results in any difference in early or late patient mortality.

\section{METHODS}

An institutional review board approved the retrospective review of adult patients undergoing cardiac surgery requiring OCM from January 2009 to December 2014. The objectives of this study were to investigate the overall indications, perioperative characteristics, complications, and mortality of patients who undergo OCM; determine the patient factors that are associated with a prolonged requirement for OCM; and investigate the potential relationship between the duration of OCM with operative and long-term mortality. Patient data were obtained from a prospectively maintained institutional clinical database and from chart reviews. Society of Thoracic Surgeons database definitions were used to define the baseline patient characteristics and complications reported in this study.
}

\section{Perioperative Management of the Incision and Antibiotic Prophylaxis}

Preoperative preparation of all cardiac surgery patients at our institution involved a chlorhexidine (4\%) scrub shower the evening before and the morning of the procedure. All surgical sites were prepped with iodine povacrylex (Duraprep; 3M, St Paul, Minn). If OCM was required, the chest cavity was irrigated with antibiotic solution and a 32 to 36 French thoracostomy tube was usually placed in addition to 2 standard mediastinal drains to ensure satisfactory mediastinal drainage. Mediastinal structures were protected from the free edge of the sternum with surgical gauze and the skin was then closed over the open chest with a running nonabsorbable suture (Figure 1) and covered with a silver-impregnated sterile dressing, which was left in place until the time of DSC or if mediastinal re-exploration was required. In procedures where skin closure was not possible due to significant mediastinal edema, either a Gore-Tex patch (W.L. Gore \& Associates, Flagstaff, Ariz) or an Esmarch bandage (Medline Industries, Mundelein, Ill) was sewn to the skin edge to achieve sterile mediastinal isolation. A video of our technique is available online (Video 1). All patients testing negative for methicillin-resistant Staphylococcus aureus were administered cefazolin before the incision. Vancomycin was added to the regimen if patients were found to be positive for methicillin-resistant $S$ aureus. In patients undergoing primary sternal closure, antibiotic prophylaxis was continued for 24 hours postoperatively. For patients requiring OCM, prophylaxis was continued until 24 hours after DSC was achieved, and was only discontinued if there was no clinical evidence of an ongoing infectious process.

\section{OCM in Intensive Care Units and Timing of DSC}

The decision to DSC was made at the discretion of the operating surgeon and was performed if all efforts to normalize severe postcardiotomy coagulopathy, hemodynamic instability, and pulmonary dysfunction were unsuccessful, or if there was a requirement for additional temporary mechanical support that would preclude primary sternal closure. All patients requiring OCM were mechanically ventilated and appropriately sedated in an intensive care unit. Mediastinal re-explorations were performed only if clinically indicated. The decision to close the chest was made by the operating surgeon and was based on resolution of the initial indication for OCM, accounting for patient hemodynamics, inotropic requirements, mediastinal drain outputs, and volume status. In patients requiring temporary circulatory support, evidence (or the absence) of myocardial recovery guided decision making on definitive therapy and ultimately timing of DSC.

\section{Statistical Analysis}

Patient demographic characteristics, perioperative characteristics, and postoperative complications during OCM were reported using percentages for categorical variables. Normality of data was assessed using the Shapiro-Wilk test and medians were used to report the central tendency of nonnormal continuous variables. Comparisons with nonnormal continuous variables were performed using the Mann-Whitney $U$ test or simple linear regression, whereas comparisons with categorical variables were performed using the $\chi^{2}$ test. Multivariable Poisson regression was used to examine the association between perioperative factors and the duration of OCM using variables with a $P$ value $\leq .1$ on bivariate analysis. Similarly, to determine the association between the duration of OCM and operative mortality, variables with a $P$ value $\leq .1$ on bivariate analysis for 30-day mortality were included in a multivariable logistic regression model to analyze for independent factors associated with this outcome. A Cox proportional-hazards model was also used to assess clinical factors that were associated with long-term survival by using variables with a $P$ value of $\leq .1$ determined by Kaplan-Meier analysis. Passive follow-up for survival status was conducted until January 2017 and this was assessed by cross-referencing evidence of patient activity in the medical record with 


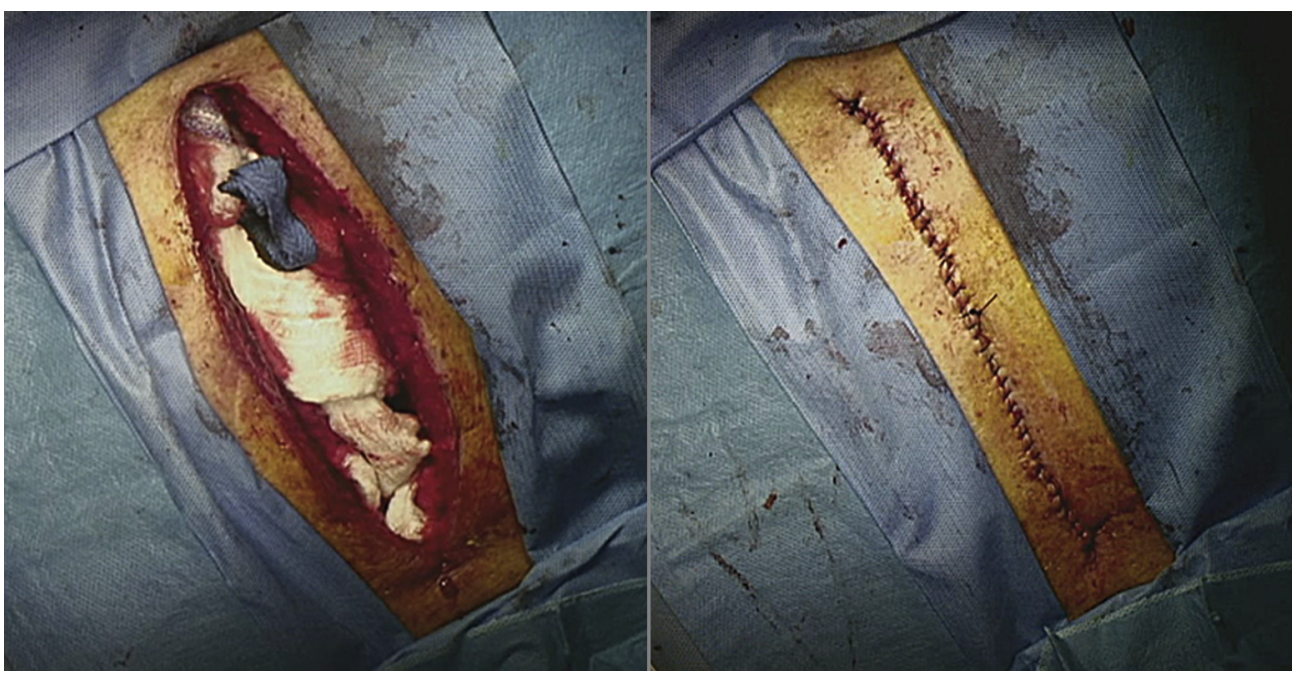

FIGURE 1. Open chest management technique for mediastinal structure isolation and protection.

the social security death index. Statistical analyses were performed using SAS version 9.3 (SAS Institute Inc, Cary, NC).

\section{RESULTS}

During the study period, 3779 cardiac surgeries were performed, of which 201 cases $(5.3 \%)$ required OCM and were included in this review. The most common surgical procedures requiring this intervention were long-term LVAD implants in 74 patients $(36.8 \%)$ followed by aortic procedures in 45 patients $(22.4 \%)$. Severe coagulopathy and hemodynamic instability represented the most common primary indication to DSC in $142(70.7 \%)$ and $21(10.5 \%)$ patients, respectively, whereas the need for central extracorporeal membrane oxygenation precluding primary sternal closure in 9 patients $(4.5 \%)$ was the least frequent indication for OCM. The remaining procedures requiring, and indications for, OCM are summarized in Table 1.

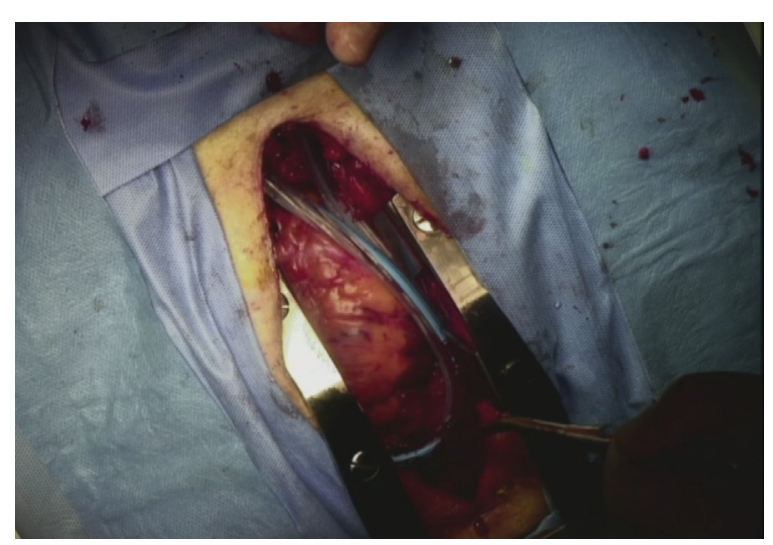

VIDEO 1. University of Rochester Medical Center delayed sternal closure technique. Video available at: http://www.jtcvsonline.org/article/S00225223(17)30704-3/addons.

\section{Factors Associated With Duration of OCM}

The median age of the overall OCM cohort was 62 years and 137 patients $(68.2 \%)$ were men. The overall median duration of OCM in this study was 3 days. Perioperative factors that were observed more frequently in patients who required prolonged OCM are summarized in Table 2. Increasing age was not associated with a longer requirement for OCM $(P=.24)$, whereas men required significantly lengthier periods of this intervention $(P=.005)$. Median OCM duration was also noted to be significantly longer when variables indicative of significant preoperative cardiac dysfunction were present; these included preoperative cardiopulmonary resuscitation (CPR) (median, 9 vs 2 days; $P=.003$ ), cardiogenic shock

TABLE 1. Procedures requiring open chest management (OCM) and its indications

\begin{tabular}{lc}
\hline \multicolumn{1}{c}{ Procedure } & OCM, $\mathbf{N}=\mathbf{2 0 1}$ \\
\hline Index procedure & \\
Ventricular assist device implant & $74(36.8)$ \\
Aortic procedure & $45(22.4)$ \\
Valve procedure & $24(11.9)$ \\
OHT & $20(10.0)$ \\
CABG & $15(7.4)$ \\
CABG/valve & $13(6.5)$ \\
Total artificial heart implant & $5(2.5)$ \\
Other & $5(2.5)$ \\
Indication & \\
Severe coagulopathy & $142(70.7)$ \\
Hemodynamic instability & $21(10.5)$ \\
Right ventricle dysfunction & $16(8.0)$ \\
Severe pulmonary failure & $13(6.5)$ \\
Central ECMO & $9(4.5)$ \\
\hline
\end{tabular}

Values are presented as n (\%). OCM, Open chest management; $O H T$, orthotopic heart transplant; $C A B G$, coronary artery bypass graft; $E C M O$, extracorporeal membrane oxygenation. 
TABLE 2. Perioperative factors associated with duration of open chest management (OCM)

\begin{tabular}{|c|c|c|c|c|}
\hline \multirow[b]{2}{*}{ Characteristic } & \multirow[b]{2}{*}{ OCM, $\mathbf{N}=201$} & \multicolumn{2}{|c|}{ Median OCM duration (Q1-Q3) } & \multirow[b]{2}{*}{$P$ value } \\
\hline & & Without variable & With variable & \\
\hline Age $(y)^{*}$ & $62(50-69)$ & \multicolumn{2}{|c|}{$R^{2}=0.007$} & .24 \\
\hline Male & $137(68.2)$ & $2(1-4)$ & $3(2-7)$ & .005 \\
\hline Body mass index* & $29(25-33)$ & \multicolumn{2}{|c|}{$R^{2}=0.014$} & .09 \\
\hline Current smoker & $24(11.9)$ & $3(2-6)$ & $4(2-7)$ & .09 \\
\hline Diabetes mellitus & $56(27.9)$ & $2(3-6)$ & $2(2-3)$ & .01 \\
\hline Hypertension & $137(68.2)$ & $3(2-5)$ & $3(2-6)$ & .41 \\
\hline Chronic renal insufficiency & $35(17.4)$ & $3(2-6)$ & $3(2-6)$ & .07 \\
\hline Cardiogenic shock & $74(36.8)$ & $2(2-4)$ & $5(3-8)$ & .04 \\
\hline Preoperative cardiopulmonary resuscitation & $11(5.5)$ & $2(3-6)$ & $9(6-12)$ & .003 \\
\hline Preoperative LVEF $\leq 30 \%$ & $111(55.2)$ & $2(2-4)$ & $4(2-7)$ & $<.001$ \\
\hline Redo sternotomy & $81(40.3)$ & $3(2-5)$ & $3(2-7)$ & .05 \\
\hline Emergent status & $78(38.9)$ & $3(2-5)$ & $4(2-7)$ & .001 \\
\hline Cardiopulmonary bypass time $(\mathrm{min})^{*}$ & $175(115-240)$ & \multicolumn{2}{|c|}{$R^{2}=0.011$} & .01 \\
\hline Aortic crossclamp time (min)* & $134(92-193)$ & \multicolumn{2}{|c|}{$R^{2}=0.071$} & .003 \\
\hline Intraoperative blood products (units)* & $2(1-6)$ & \multicolumn{2}{|c|}{$R^{2}=0.008$} & .19 \\
\hline Need for temporary assist device & $48(23.4)$ & $2(2-4)$ & $7(4-11)$ & $<.001$ \\
\hline Sternal re-exploration before DSC & $60(29.9)$ & $2(2-4)$ & $6(3-11)$ & $<.001$ \\
\hline \multicolumn{5}{|l|}{ Postoperative complications and mortality } \\
\hline Deep sternal wound infection & $6(3.0)$ & $2(2-6)$ & $6(2-10)$ & .51 \\
\hline Pneumonia & $19(9.5)$ & $3(2-5)$ & $11(5-19)$ & $<.001$ \\
\hline Bacteremia & $5(2.5)$ & $3(2-6)$ & $12(4-17)$ & .01 \\
\hline Sepsis & $10(5.0)$ & $3(2-6)$ & $12(7-21)$ & $<.001$ \\
\hline Renal failure & $35(17.4)$ & $3(2-5)$ & $6(3-12)$ & $<.001$ \\
\hline Acute limb ischemia & $17(8.5)$ & $3(2-6)$ & $5(3-9)$ & .02 \\
\hline Permanent cerebrovascular accident & $14(7.0)$ & $3(2-6)$ & $6(3-11)$ & .01 \\
\hline Cardiac arrest & $12(6.0)$ & $3(2-6)$ & $5(1-7)$ & .43 \\
\hline Atrial fibrillation & $39(19.4)$ & $3(2-5)$ & $4(2-7)$ & .01 \\
\hline Multisystem organ failure & $19(9.5)$ & $3(2-5)$ & $10(6-19)$ & $<.001$ \\
\hline 30-d mortality & $32(15.9)$ & $3(2-5)$ & $6(2-11)$ & .02 \\
\hline
\end{tabular}

Values are presented as median (1st quartile-3rd quartile) or n (\%). OCM, Open chest management; $L V E F$, left ventricular ejection fraction; $D S C$, delayed sternal closure. *Simple linear regression was used to measure associations.

(median, 5 vs 2 days; $P=.04$ ), and a left ventricular ejection fraction (LVEF) $\leq 30 \%$ (median, 4 vs 2 days; $P<.001)$. Other perioperative factors that were significantly associated with a longer requirement for OCM were emergency surgeries $(P<.001)$, increasing cardiopulmonary bypass $(P=.01)$, higher intraoperative blood product transfusion rates $(P=.008)$, the need for temporary mechanical support (7 vs 2 days; $P<.001)$, and sternal re-exploration before DSC (6 vs 2 days; $P<.001)$. Prolonged OCM was also significantly associated with an increased rate of complications that developed while the chest was open. Apart from deep sternal wound infections (DSWIs) $(P=.51)$, which were not found to increase in frequency with prolonged durations of OCM, pneumonias $(P<.001)$, bacteremia $(P=.01)$, and sepsis $(P<.001)$, were observed in patients who required longer OCM on bivariate analysis, with median durations of 11 , 12 , and 12 days, respectively. Postoperative renal failures were among the most prevalent complications noted during OCM and were also significantly associated with longer durations of this intervention ( 6 vs 3 days; $P<.001$ ).
On multivariable Poisson regression, pneumonias, sternal re-explorations, and the requirement for a temporary mechanical assist device, when present, were each independently associated with a $>50 \%$ longer duration of OCM days $(P<.001)$. Renal failure (incidence rate ratio [IRR], 1.28; 95\% confidence interval [CI], 1.03-1.60), male sex (IRR, 1.35; 95\% CI, 1.14-1.60), and preoperative LVEF $\leq 30 \%$ (IRR, 1.36; 95\% CI, 1.16-1.61) were also independently associated with a longer duration of OCM. The remaining results from the multivariable analysis are detailed in Table 3.

\section{Factors Associated With 30-day and Long-Term Mortality in Patients Undergoing OCM}

Of the 201 patients who underwent OCM, 169 (84.1\%) were alive at 30 days postoperatively, whereas 32 experienced mortalities $(15.9 \%)$. The 30-day mortality rate by procedure was highest in patients undergoing total artificial heart implant (4 out of 5 patients; 80\%), followed by coronary artery bypass grafting ( 5 out of 15 patients; $33 \%$ ), valve cases (6 out of 24 patients; $25 \%$ ), aortic 
TABLE 3. Poisson regression analysis assessing factors associated with duration of open chest management $(\mathrm{OCM})$

\begin{tabular}{lcc}
\hline \multicolumn{1}{c}{ Factor } & $\begin{array}{c}\text { Incidence rate ratio } \\
(\mathbf{9 5} \% \text { confidence interval })\end{array}$ & $\begin{array}{c}\boldsymbol{P} \\
\text { value }\end{array}$ \\
\hline Male & $1.35(1.14-1.60)$ & $<.001$ \\
Chronic renal insufficiency & $1.19(0.98-1.44)$ & .07 \\
Preoperative LVEF $\leq 30 \%$ & $1.36(1.16-1.61)$ & $<.001$ \\
Need for temporary assist device & $1.57(1.32-1.86)$ & $<.001$ \\
Pneumonia & $1.63(1.30-2.04)$ & $<.001$ \\
Sepsis & $1.25(0.96-1.63)$ & .10 \\
Postoperative renal failure & $1.28(1.03-1.60)$ & .02 \\
Sternal re-exploration before DSC & $1.62(1.37-1.91)$ & $<.001$ \\
Multi-system organ failure & $1.34(1.09-1.65)$ & .007 \\
Atrial fibrillation & $1.26(1.04-1.52)$ & .02 \\
\hline
\end{tabular}

$L V E F$, Left ventricular ejection fraction; $D S C$, delayed sternal closure.

procedures ( 7 out of 45 patients; $16 \%$ ), combined coronary artery bypass grafting with a valve case ( 2 out of 13 patients; $15 \%$ ), orthotopic heart transplants ( 2 out of 20 patients; $10 \%$ ), and was lowest in patients undergoing LVAD implants (6 out of 74 patients; $8 \%$ ). Perioperative factors that were significantly associated with 30-day mortality on bivariate analysis included the need for emergency surgery $(56 \%$ vs $36 \% ; P=.03)$, sternal re-exploration ( $53 \%$ vs $25 \% ; P=.001)$, and the duration of OCM (median 6 days in nonsurvivors vs 3 days in survivors; $P=.03$ ). Increasing age, hypertension, cardiogenic shock, preoperative CPR, and redo sternotomies all trended toward a significant association with operative mortality $(P \leq .10)$ (Table 4$)$ and these variables were included in a multivariable logistic regression model. The results from the multivariable analysis revealed that the odds of operative mortality independently increased by $5 \%$ for every yearly increment in age $(P=.01)$; this effect was more pronounced when analyzing the duration of OCM, which resulted in an $11 \%$ increased odds of 30-day mortality for every day sternal closure was delayed $(P=.01)$. The other variables selected for inclusion in this model were not found to be significant and are summarized in Table 5.

Follow-up was completed in $94 \%$ of survivors at a mean of $50 \pm 24$ months with an overall survival of $51 \%$. A Cox proportional-hazards model was then used to assess the association between perioperative factors with long-term mortality and the results from this analysis are presented in Table 6. Notably, the duration of OCM was found to be independently associated with a $9 \%$ increased risk of

TABLE 4. Bivariate analysis of factors associated with 30-day mortality

\begin{tabular}{|c|c|c|c|}
\hline Characteristic & Survivors at $30 \mathrm{~d}, \mathrm{n}=169$ & Nonsurvivors at $30 \mathrm{~d}, \mathrm{n}=32$ & $P$ value \\
\hline Age (y) & $62(48-69)$ & $63.0(55-72)$ & .08 \\
\hline Male & $113(66.9)$ & $24(75.0)$ & .41 \\
\hline Body mass index & $29(25-32)$ & $29(26-35)$ & .32 \\
\hline Current smoker & $20(11.8)$ & $4(12.5)$ & 1.0 \\
\hline Diabetes mellitus & $46(27.2)$ & $10(31.3)$ & .65 \\
\hline Hypertension & $111(65.5)$ & $26(81.3)$ & .10 \\
\hline Chronic renal insufficiency & $26(15.4)$ & $9(28.1)$ & .12 \\
\hline Cardiogenic shock & $29(24.8)$ & $17(53.1)$ & .05 \\
\hline Preoperative cardiopulmonary resuscitation & $7(4.2)$ & $4(12.5)$ & .08 \\
\hline Preoperative $\mathrm{LVEF} \leq 30 \%$ & $96(56.8)$ & $15(46.9)$ & .34 \\
\hline Redo sternotomy & $3(2-5)$ & $3(2-7)$ & .05 \\
\hline Emergent status & $60(35.5)$ & $18(56.3)$ & .03 \\
\hline Cardiopulmonary bypass time (min) & $175(119-239)$ & $157(106-256)$ & .80 \\
\hline Aortic crossclamp time (min) & $134(95-189)$ & $127(88-209)$ & .96 \\
\hline Intraoperative blood products (units) & $5(2-11)$ & $5(2-15)$ & .26 \\
\hline Need for temporary assist device & $38(22.5)$ & $10(31.3)$ & .36 \\
\hline Sternal re-exploration before DSC & $43(25.4)$ & $17(53.1)$ & .001 \\
\hline Open chest duration & $3(2-5)$ & $6(2-11)$ & .03 \\
\hline \multicolumn{4}{|l|}{ Postoperative complications } \\
\hline Deep sternal wound infection & $3(1.8)$ & $1(3.1)$ & .50 \\
\hline Pneumonia & $13(7.7)$ & $6(18.8)$ & .09 \\
\hline Bacteremia & $4(2.4)$ & $1(3.1)$ & .58 \\
\hline Sepsis & $7(4.1)$ & $3(9.4)$ & .20 \\
\hline Renal failure & $19(11.2)$ & $16(50.0)$ & $<.001$ \\
\hline Acute limb ischemia & $12(7.1)$ & $5(15.6)$ & .16 \\
\hline Permanent cerebrovascular accident & $8(4.7)$ & $6(18.8)$ & .12 \\
\hline Cardiac arrest & $7(4.1)$ & $5(15.6)$ & .03 \\
\hline Atrial fibrillation & $31(18.3)$ & $8(25.0)$ & .46 \\
\hline Multisystem organ failure & $6(3.6)$ & $13(40.6)$ & .09 \\
\hline
\end{tabular}

Values are presented as median (1st quartile-3rd quartile) or $\mathrm{n}(\%) . L V E F$, Left ventricular ejection fraction; DSC, delayed sternal closure. 
TABLE 5. Multivariable regression analysis assessing factors associated with 30-day mortality

\begin{tabular}{|c|c|c|}
\hline Factor & $\begin{array}{c}\text { Odds ratio } \\
\text { (95\% confidence } \\
\text { interval) }\end{array}$ & $\begin{array}{c}P \\
\text { value }\end{array}$ \\
\hline Age & $1.05(1.01-1.10)$ & .01 \\
\hline Hypertension & $1.67(0.59-4.76)$ & .34 \\
\hline Preoperative cardiopulmonary resuscitation & $0.62(0.13-3.14)$ & .55 \\
\hline Preoperative cardiogenic shock & $2.22(0.71-6.93)$ & .17 \\
\hline Redo sternotomy & $1.96(0.82-4.66)$ & .13 \\
\hline Emergent status & $2.12(0.75-6.04)$ & .15 \\
\hline OCM duration (d) & $1.11(1.02-1.21)$ & .01 \\
\hline Sternal re-exploration before DSC & $1.65(0.61-4.41)$ & .32 \\
\hline
\end{tabular}

$O C M$, Open chest management; $D S C$, delayed sternal closure.

4-year mortality for every additional day this intervention was required $(P<.001)$. Interestingly, chronic renal insufficiency was also found to be associated with an increased risk for late mortality $(P=.005)$, while increasing age trended toward a significant effect on this outcome $(P=.08)$.

\section{DISCUSSION}

Open chest management with DSC began in the late 1970s and its use expanded after early studies demonstrated acceptable survival rates and a low incidence of DSWIs, which had represented a major obstacle to widespread acceptance. The survival in these early studies ranged from $48 \%$ to $66 \%$ of patients and the incidence of DSWIs were reported to be between $2 \%$ and $4 \%{ }^{4,5,10,11}$ More recently, the outcomes of OCM patients have improved, with several studies reporting survival as high as $76 \%$ to $89 \%,{ }^{2,3,12}$ likely occurring as a result of improved perioperative patient selection and advancements in critical care. OCM in a contemporary cohort of patients at our institution was required in $5 \%$ of patients undergoing cardiac surgery, resulting in a survival rate of $84 \%$, which is similar to other reports in the literature. ${ }^{3,5,7,13}$

In this study, a longer duration of OCM was significantly associated with an increased likelihood of 30-day mortality. This finding was noted previously by Anderson and colleagues $^{2}$ in a study of 87 patients undergoing OCM.

TABLE 6. Cox proportional-hazards analysis assessing factors associated with 4-year mortality

\begin{tabular}{lcc}
\hline \multicolumn{1}{c}{ Factor } & $\begin{array}{c}\text { Hazard ratio (95\% } \\
\text { confidence interval) }\end{array}$ & $\begin{array}{c}\boldsymbol{P} \\
\text { value }\end{array}$ \\
\hline Age & $1.02(1.00-1.04)$ & .08 \\
Current smoker & $0.49(0.17-1.13)$ & .13 \\
Chronic renal insufficiency & $2.08(1.23-3.40)$ & .005 \\
Hypertension & $1.46(0.88-2.54)$ & .16 \\
Redo sternotomy & $1.43(0.91-2.23)$ & .12 \\
OCM duration (d) & $1.09(1.04-1.13)$ & $<.001$ \\
Sternal re-exploration before DSC & $0.87(0.49-1.50)$ & .63 \\
\hline
\end{tabular}

$O C M$, Open chest management; $D S C$, delayed sternal closure.
They report an average time of 6.2 days in patients who experienced operative mortalities versus 3.2 days in survivors. Boeken and colleagues ${ }^{3}$ report a similar finding with mortalities noted at an average of 6.4 days in 212 patients with an open chest (vs 3.2 days), and in their analysis a delay of $>6$ days before sternal closure was found to be independently associated with a 2.6 times increased risk of death. We also observed a median time of 6 days with an open chest in nonsurvivors compared with 3 days in survivors and found that this risk of early mortality increased by $11 \%$ for each day sternal closure was delayed. Furthermore, such a delay was also found to increase the risk of late mortality by $9 \%$, providing more evidence as to its association with poor patient outcomes. Notably, quantification of the risk to delaying sternal closure has not been performed to date and our findings remained significant despite controlling for potential factors that could confound this outcome such as preoperative low LVEF, cardiogenic shock, CPR, and emergent status. It is possible that this increased mortality rate observed with prolonged durations of OCM was reflective of the severity of illness of the patient, resulting from the insult of surgery on a largely precarious clinical baseline; however, it is also likely that the act of delaying sternal closure for a substantial period increases the risk of complications, particularly those of infectious origin, which was observed in our study and could contribute to the poorer outcomes noted in these patients. These results, in addition to others provided in the literature, emphasize the importance of infection prevention and early sternal closure in patients undergoing OCM whenever possible.

Although an understanding of the influence of prolonged OCM on mortality can be valuable when weighing the risks and benefits of earlier sternal closure, knowledge of the perioperative factors that are associated with such a requirement may be just as important. Among these factors, sternal re-explorations, and variables that were indicative of severe cardiac dysfunction either preoperatively (LVEF $\leq 30 \%$ ) or immediately postoperatively (requirement for temporary mechanical support) were independently associated with the need for prolonged durations of OCM on multivariable regression. To our knowledge, no study has performed such an analysis, which limits our review of available data on these findings; however, Anderson and colleagues ${ }^{2}$ note that re-explorations for bleeding and the requirement for a temporary ventricular assist device were both predictive of mortality in their study, which they also associate with a prolonged time to DSC. Infectious complications significantly increased in frequency with longer durations of OCM-pneumonias and sepsis, specifically, were independent factors for this outcome and when present, were diagnosed at approximately 12 days of OCM. DSWIs did not occur more frequently with increasing time of OCM 
in our analyses, which suggests that the reported increased risk of developing DSWIs with $\mathrm{OCM}^{2,3}$ could be unrelated to the duration the chest is left open; however, due to the low incidence of this complication, our study may not be adequately powered to demonstrate such a difference and thus only limited conclusions can be made from this finding. Ultimately, these results highlight the importance of infection prevention in the care of these severely critical patients who are ill placed to tolerate an infectious burden in addition to the stressors of surgery and their underlying disease process. These results provide further insight into the factors associated with prolonged OCM, which were previously poorly understood and will be important in the preoperative identification and postoperative management of patients who are at an increased likelihood of requiring extended periods of this intervention after cardiac surgery.

Renal complications were a major source of morbidity experienced by OCM patients and have been reported to affect approximately $16 \%$ to $30 \%$ of cases in similar studies. ${ }^{3,5,14}$ In our review, renal failure developed in $17 \%$ of patients during OCM and was found to be an independent risk factor for a prolonged need for this intervention. Furnary and colleagues, ${ }^{4}$ in their review of 107 patients undergoing OCM, found that sternal closure was more likely if a negative fluid balance was achieved for $>48$ hours, noting that a marked diuresis would assist in reducing myocardial edema and thus improving the likelihood of successful DSC; they also report an improved prognosis if renal function is preserved, which is likely due to the patients' own capacity to restore an euvolemic state. Their findings correlate with the results in this study because the presence of renal failure, which was defined as either an increase in serum creatinine level 3.0 times greater than baseline, $\geq 4 \mathrm{mg} / \mathrm{dL}$, or a new requirement for dialysis, was significantly more prevalent in patients who experienced mortalities $(50 \%)$ compared with those who did not $(11 \%)(P<.001)$, and was independently associated with a $23 \%$ longer duration of OCM days. Additionally, multiple series have identified renal failure or the need for dialysis as significant risk factors for a poor outcome in OCM patients, ${ }^{2-4,14}$ highlighting the importance of renal-protective strategies in this population to preserve and maintain renal function whenever possible. These involve, among others, the perioperative optimization of hemodynamic parameters, adequate resuscitation to maintain intravascular volume, avoidance or cautious use of nephrotoxic agents, and early identification of renal injury to prevent progression to failure.

In summary, the results from this study provide further evidence that OCM in a contemporary cohort of patients can be safely performed with acceptable early and late outcomes, and remains an important therapeutic intervention in patients who are unable to undergo safe primary sternal closure following cardiac surgery. Despite this, the increased early and late mortality rate, and higher perioperative morbidity noted in patients who require prolonged durations of OCM, emphasize the importance of closing the chest expeditiously by continuously assessing if ongoing OCM is needed, and also support a high degree of vigilance for associated complications, particularly those of infectious and renal origin.

\section{Limitations}

There are several limitations associated with this study. We had difficulty retrospectively determining the primary reason to delay sternal closure in a number of patients because the decision was often multifactorial and involved a combination of hemodynamic instability, coagulopathy, and use of temporary assist devices. This decision-making process, or descriptions of attempts to close the sternum primarily before deciding to delay sternal closure, was not consistently documented in operative notes. Delays in chest closure could have been related to extramural factors, such as operating room availability, surgeon coverage, weekend schedule, and patient neurological status, which were difficult to retrospectively analyze but represent an important factor to account for when managing OCM patients. These likely represent an area in which significant improvements in the processes of care can be made.

\section{Webcast}

You can watch a Webcast of this AATS meeting presentation by going to: http://webcast.aats.org/2016/Video/Monday/ 05-16-16_Room_337_1500_Wong-800.mp4.

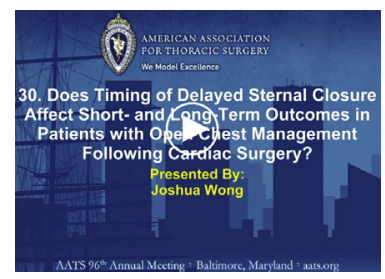

\section{Conflict of Interest Statement}

J.K.W., D.J.J., A.L.M., and P.A.K. have received grant support from LSI Solutions, Victor, NY, for research unrelated to this study. H.T.M. is a proctor for Thoratec Corporation. All other authors have nothing to disclose with regard to commercial support.

\section{References}

1. Riahi M, Tomatis LA, Schlosser RJ, Bertolozzi E, Johnston DW. Cardiac compression due to closure of the median sternotomy in open heart surgery Chest. 1975;67:113-4.

2. Anderson CA, Filsoufi F, Aklog L, Farivar RS, Byrne JG, Adams DH. Liberal use of delayed sternal closure for postcardiotomy hemodynamic instability. Ann Thorac Surg. 2002;73:1484-8.

3. Boeken U, Assmann A, Mehdiani A, Akhyari P, Lichtenberg A. Open chest management after cardiac operations: outcome and timing of delayed sterna closure. Eur J Cardiothorac Surg. 2011;40:1146-50. 
4. Furnary A, Magovern J, Simpson K, Magovern G. Prolonged open sternotomy and delayed sternal closure after cardiac operations. Ann Thorac Surg. 1992; 54:233-9.

5. Christenson JT, Maurice J, Simonet F, Velebit V, Schmuziger M. Open chest and delayed sternal closure after cardiac surgery. Eur J Cardiothorac Surg. 1996;10: 305-11.

6. Shalabi RIY, Amin M, Ayed AK, Shuhiber H. Delayed sternal closure is a life saving decision. Ann Thorac Cardiovasc Surg. 2002;8:220-3.

7. Stulak JM, Romans T, Cowger J, Romano MA, Haft JW, Aaronson KD, et al. Delayed sternal closure does not increase late infection risk in patients undergoing left ventricular assist device implantation. J Hear Lung Transplant. 2012;31:1115-9.

8. Johnson J, Gundersen E, Stickney I, Cogbill T. Selective approach to sternal closure after exploration for hemorrhage following coronary artery bypass. Ann Thorac Surg. 1990;49:771-4.

9. Josa M, Khuri SF, Braunwald NS, VanCisin MF, Spencer MP, Evans DA, et al. Delayed sternal closure. An improved method of dealing with complications after cardiopulmonary bypass. J Thorac Cardiovasc Surg. 1986;91:598-603.

10. Freeman RK, Daily PO, Dembitsky WP, Adamson RM, Moreno-Cabral RJ. The treatment of low cardiac output syndrome following cardiopulmonary bypass using delayed sternal closure. Am Surg. 1997;63:882-4.

11. Fanning WJ, Vasko JS, Kilman JW. Delayed sternal closure after cardiac surgery. Ann Thorac Surg. 1987;44:169-72.

12. Stulak JM, Dearani JA, Burkhart HM, Barnes RD, Scott PD, Schears GJ. ECMO cannulation controversies and complications. Semin Cardiothorac Vasc Anesth. 2009; 13:176-82.

13. Spiliopoulos S, Autschbach R, Koerfer R, Tenderich G. Delayed sternal closure after total artificial heart implantation. J Thorac Cardiovasc Surg. 2015;150: 417-8.

14. Hashemzadeh K, Hashemzadeh S. In-hospital outcomes of delayed sternal closure after open cardiac surgery. J Card Surg. 2009;24:30-3.

Key Words: open chest management, delayed sternal closure, long-term survival, postoperative complications, clinical outcomes

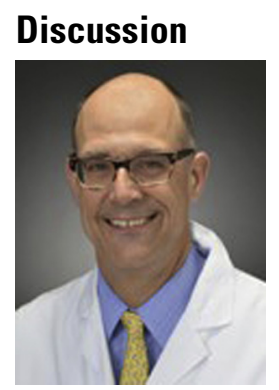

Dr Bruce J. Leavitt (Burlington, Vt). I'd like to thank the Association for the opportunity to discuss this information and Dr Wong and his colleagues from the University of Rochester for getting the manuscript to me in a timely fashion. One of the last slides was changed a little bit, so now you've got 3 questions instead of 4 because you answered the fourth one.

This is the most detailed study of a rare situation that cardiac surgeons encounter: When you get in there, you do a tough case, and you cannot close the chest. There is not a lot of literature on this subject, so I applaud the authors for tackling something and providing a very detailed report.

First, the published rate of management for open closure or delayed closure ranges from $1.8 \%$ to $4 \%$ in all the published studies in your reference list that I read.

Our rate at our hospital is clearly not $5 \%$, and your rate is closer to $5 \%$. From your manuscript, you say that $70 \%$ of your open chest patients have severe coagulopathy. In your talk, you said it was a mixture. But in your description you say $70 \%$ of the reason for open chest management is coagulopathy and $10 \%$ was hemodynamic instability.

Was there a consideration of longer time in the operating room with correction of coagulopathy and then a standard closure? Our acceptance standard for re-exploration is between $1 \%$ and $2 \%$, so my question is, have you considered staying in the operating room longer, correcting a coagulopathy, and then trying to close?

Dr Wong. Thank you for your question and for reviewing our work.

Firstly, severe coagulopathy was the primary indication for open chest management in $70 \%$ of our patients based on documentation in the operative notes. There were patients where more than 1 indication existed that we determined from postoperative clinical notes; however, we report only the primary indication to avoid confusion.

As we mentioned, a lot of the cases that required open chest management were cases where the surgeon actually did stay in the operating room for as long as possible with the intention to correct the coagulopathy. However, because of the time that was taken and the severity of the coagulopathy, it was determined that open chest management would be better in these group of patients.

Additionally, a significant number of our patients had a left ventricular assist device, and the literature in those group of patients supports a more liberal use of this intervention, so I think the rate of coagulopathy that was reported was probably influenced by that.

Dr Leavitt. My second question is 23\% of your patients had temporary circulatory support. None of your patients in the control center had external support. Is that a standard protocol for your institution?

Dr Wong. Yes. So at our institution, we were fairly liberal over the past 5 years with regard to the implantation of CentriMag ventricular assist devices for temporary right and left heart support. That practice has somewhat changed slightly where we are attempting to go with more percutaneous interventions for support, and therefore we have not been as liberal with regard to open chest management in patients who required temporary circulatory support.

Dr Leavitt. Now, my final question because the fourth one is you didn't document the types of procedures, but in your slide you did.

So my last question is about patients with preoperative cardiopulmonary resuscitation (CPR) who had 16 times the rate of open chest management compared with those without preoperative CPR. In our institution, patients do not go to the operating room unless CPR has been discontinued, and hemodynamic stability or somewhat stability has occurred. 
Could you further describe your institution's practice on preoperative CPR and cardiogenic shock, and when do you go to the operating room for emergency surgery?

Dr Wong. Thank you. With regard to CPR and cardiogenic shock, we do our best to resolve or stabilize the hemodynamic instability that they present with before surgery, similar to most institutions. With regard to CPR, that was a variable that was coded in the Society of Thoracic Surgeons database, and although we reviewed it ourselves, it was hard to determine whether CPR was ongoing at the time of surgery or prior. Because our practice was to hemodynamically stabilize patients before emergency surgery, instances where CPR occurred at the time of surgery were likely rare.

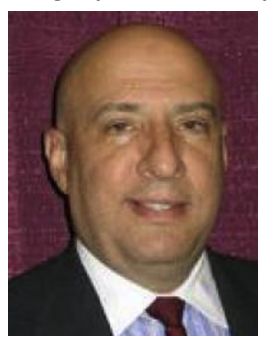

Dr Darryl M. Hoffman (Elmira, $N Y$ ). I enjoyed your talk. A couple of my patients have ended up in your hands, so thank you.

A question for you. What protocol do you apply for the practical management of an open chest patient? What do you do about washing them out? Do you try and close the skin? Do you use a temporary membrane barrier? Please let us know.

Dr Wong. Thank you for your question. Our protocol for open chest management is to close the skin over the open sternum whenever possible. When that is not anatomically possible, we use the Esmarch bandage or a polytetrafluoroethylene patch, although those instances are rare.

With regard to washouts, they are not routinely performed, and we would only do them if clinically indicated or if open chest management was required for an extended duration, which would be between 5 to 6 days. But they were not routinely performed.

And with regard to antibiotic prophylaxis, we continue these patients on cefazolin for the perioperative period until delayed sternal closure unless there is evidence of any infectious process going on.

Dr Hoffman. I have to push back a little. In $70 \%$ of your patients with coagulopathy you closed the skin or you put in a membrane in. Do you not go back in and wash them out? You are leaving people who are effectively pretamponade, and you may get away with it a lot because you are using mechanical devices.

Dr Wong. I forgot to mention that we do leave an additional mediastinal drain, and it is a fairly large chest tube that we leave in place in addition to 2 other mediastinal tubes.

And we do close the skin and watch their coagulopathy or their drainage outputs over the immediate perioperative period.

So we do not believe that increases the risk of tamponade, if that was your question.
Dr Hoffman. Not really, but you are leaving a lump of warm, wet culture medium in the mediastinum for 5 to 7 days with foreign material as well. Thank you.

Dr Wong. I apologize. Although routine washouts were not performed at an earlier time period, if clinically indicated, patients would be brought back to the operating room for re-explorations or washouts and that was at the surgeons' discretion. Furthermore, not every patient with coagulopathy required open chest management for a prolonged duration. Lastly, we sampled and analyzed mediastinal cultures in all patients undergoing open chest management and did not find an increased rate of positive cultures in patients requiring prolonged open chest management.

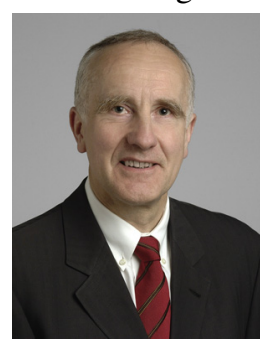

Dr Gosta B. Pettersson (Cleveland, Ohio). Do you ever use vacuum?

Dr Wong. Not at our institution. Although we do recognize that vacuum is something that has been clinically used.

Dr Pettersson. A second comment, I am sure that if I had gone to Dr Blackstone with this he would have had some issues in analyzing the actual contribution of the open chest and duration to the outcome.

Dr Wong. Thank you for your questions. We did deal with this issue, and it is similar to a chicken-and-egg situation. We had difficulty determining whether it was the preoperative dysfunction that resulted in the higher mortality in patients requiring longer open chest management versus the actual open chest.

I think the only thing that we can conclusively say is that preoperative cardiac dysfunction significantly contributed to poor outcomes in these patients; however, infections and renal failure also significantly increased with prolonged open chest management and could have also had an effect on mortality.

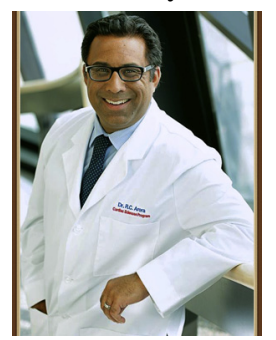

Dr Rakesh C. Arora (Winnipeg, Canada). Excellent talk. A question with regard to your ventilator-associated pneumonia bundle compliance, timing nutrition initiation, and central line access protocols for the 3 groups. Were there differences, and did that influence your postoperative infection rates?

Dr Wong. We did not specifically look at differences in the protocols that you mentioned; however, they are fairly standard. I do not think we treated them any differently.

Unfortunately, we did not investigate this, but it is a valid question and an important point that we will re-review. 


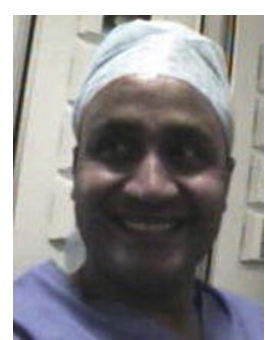

Dr S. Saeed Ashraf (Swansea, United Kingdom). Of your $70 \%$ of coagulopathic patients where you just closed the skin, do you normally pack the chest with swab, and if you do, how often do you have to go back to the operating room to take the swab out and then come back because of tamponade or because the coagulopathy was not stopping?
Dr Wong. Thank you for your question. In patients with coagulopathy, we tend to pack the chest with sponges. If there is foreign body there, then we are more likely to go back at an earlier period.

I do not have the rates of return to the operating room specifically in the category of patients who were coagulopathic, but we did not find that we were going back to the operating room any more frequently than usual because of tamponade physiology.

Readers who found these articles interesting may also like to read the following papers found in recent and future issues of our sister publications, Seminars in Thoracic and Cardiovascular Surgery and Operative Techniques in Thoracic and Cardiovascular Surgery!

Acquired: Perioperative Management

ORIGINAL SUBMISSION: The Effects of Steroids on Coagulation Dysfunction Induced by Cardiopulmonary Bypass: A Steroids in Cardiac Surgery (SIRS) Trial Substudy. Domenico Paparella. Semin Thoracic Surg 2017: In press.

Editorial Commentary: Steroids and Cardiopulmonary Bypass: a Never-ending Story. Thierry Carrel. Semin Thoracic Surg 2017: In press.

ORIGINAL SUBMISSION: Controlled Exponentially Weighted Moving Average Chart in Cardiac Surgery: A Simulation Study Across 9 Italian Cardiac Centers. Marco Moscarelli. Semin Thoracic Surg 2016:253-258.

Editorial Commentary: Statistical Process Control in Cardiac Surgery? Ask Your Doctor. Paul Kurlansky. Semin Thoracic Surg 2016:259-260.

ORIGINAL SUBMISSION: Early Extubation: A Proposed New Metric. Todd C. Crawford. Semin Thoracic Surg 2016:290-299.

Editorial Commentary: Early Extubation: A Proposed New Metric or What Was First: The Egg or the Chicken? Thierry Carrel. Semin Thoracic Surg 2016:300-301. 